\title{
The requirement for freshly isolated human colorectal cancer (CRC) cells in isolating CRC stem cells
}

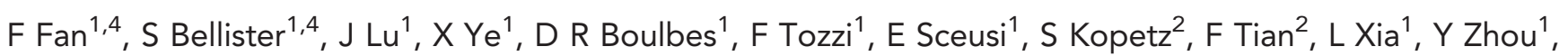
R Bhattacharya ${ }^{1}$ and L M Ellis ${ }^{*, 1,3}$

${ }^{1}$ Department of Surgical Oncology, Unit 1484, University of Texas MD Anderson Cancer Center, 1515 Holcombe Boulevard, Houston, Texas 77230-1402, USA; ${ }^{2}$ Department of Gastrointestinal Medical Oncology, University of Texas MD Anderson Cancer Center, Houston, Texas, USA and ${ }^{3}$ Department of Molecular and Cellular Oncology, University of Texas MD Anderson Cancer Center, Houston, Texas, USA

Background: Isolation of colorectal cancer (CRC) cell populations enriched for cancer stem cells (CSCs) may facilitate target identification. There is no consensus regarding the best methods for isolating CRC stem cells (CRC-SCs). We determined the suitability of various cellular models and various stem cell markers for the isolation of CRC-SCs.

Methods: Established human CRC cell lines, established CRC cell lines passaged through mice, patient-derived xenograft (PDX)-derived cells, early passage/newly established cell lines, and cells directly from clinical specimens were studied. Cells were FAC-sorted for the CRC-SC markers CD44, CD133, and aldehyde dehydrogenase (ALDH). Sphere formation and in vivo tumorigenicity studies were used to validate CRC-SC enrichment.

Results: None of the markers studied in established cell lines, grown either in vitro or in vivo, consistently enriched for CRC-SCs. In the three other cellular models, CD44 and CD133 did not reliably enrich for stemness. In contrast, freshly isolated PDX-derived cells or early passage/newly established CRC cell lines with high ALDH activity formed spheres in vitro and enhanced tumorigenicity in vivo, whereas cells with low ALDH activity did not.

Conclusions: PDX-derived cells, early passages/newly established CRC cell lines and cells from clinical specimen with high ALDH activity can be used to identify CRC-SC-enriched populations. Established CRC cell lines should not be used to isolate CSCs.

Cancer stem cells (CSCs) have been identified in multiple types of malignancies and are hypothesised to mediate resistance to therapies (Storms et al, 1999; Dalerba et al, 2007; Ginestier et al, 2007; Gaur et al, 2011; Kim et al, 2011; Lohberger et al, 2012). By definition, CSCs demonstrate pluripotency and the capacity for self-renewal (O'Brien et al, 2007; Ricci-Vitiani et al, 2007). Standard criteria used to validate CSCs include anchorageindependent growth and tumorigenicity, but standard methods for identification and validation of CSCs, especially in the field of colorectal cancer (CRC), are currently being challenged (Shmelkov et al, 2008; Wu et al, 2014). Validation of assays that enrich for the CRC stem cell (CRC-SC) population is essential in order to study CSC biology and therapies to target this cell subpopulation.

It is unclear whether CSCs can be reliably isolated from established CRC cell lines. Cell lines that have been propagated in long-term in vitro culture conditions undergo selection pressures and/or clonal dominance that yield relatively homogeneous cell populations (Hughes et al, 2007; Fredebohm et al, 2012). Given

\footnotetext{
*Correspondence: Dr LM Ellis; E-mail: lellis@mdanderson.org

${ }^{4}$ These authors contributed equally to this work.
}

Received 4 March 2014; revised 19 November 2014; accepted 26 November 2014; published online 23 December 2014 
this homogeneity, established cell lines are not representative of tumours in patients but are still being used in CSC studies that rarely describe attempts to recapitulate the cellular heterogeneity and tumoural hierarchy of human tumours (Chu et al, 2009; Yeung et al, 2010).

CSCs are most commonly identified by differential expression of unique cell surface glycoproteins; in solid malignancies, cluster of differentiation markers such as CD133 and CD44 have commonly been used to this end (Dalerba et al, 2007; O'Brien et al, 2007; Ricci-Vitiani et al, 2007; Du et al, 2008). The use of these markers to characterise CSCs is rife with controversy, including determining which marker has the highest fidelity for CSC enrichment (Shmelkov et al, 2008; Huang et al, 2009). Functional assays for isolation of CSCs enrichment provide an alternative to the use of cell surface markers. The Aldefluor assay measures the activity of several isoforms of aldehyde dehydrogenase (ALDH), whose native intracellular functions include detoxification (Canino et al, 2012; Moreb et al, 2012). The Aldefluor assay has been used extensively to identify CSCs in patient tissue samples, including CRC (Chu et al, 2009; Huang et al, 2009; Lohberger et al, 2012).

In our studies, we sought to determine the utility of established CRC cell lines, patient-derived CRC cell lines, and various stem cell markers for the isolation or enrichment of CRC-SCs. We demonstrated that established CRC cell lines, regardless of the marker utilised, should not be used for the study of CSCs. However, patient tumour-derived xenografts and the freshly CRC-derived cell lines could be used to isolate CSCs using the Aldefluor assay, but not CD133 and CD44.

\section{MATERIALS AND METHODS}

Established cell lines. The human CRC cell lines HCT116, HT29, and SW480 (American Type Culture Collection, Manassas, VA, USA) were maintained in culture using standard protocols. Cells were confirmed to be free of mycoplasma using the MycoAlert mycoplasma detection kit (Lonza Group, Allendale, NJ, USA). Cell line identity was validated by short-tandem repeat genotyping every 6 months in the Characterized Cell Line Core at the University of Texas MD Anderson Cancer Center. Results from all in vitro studies were confirmed in at least three independent experiments.

Patient-derived xenograft (PDX)-derived cells and freshly isolated cell lines. Patients undergoing primary CRC resection at the MD Anderson Cancer Center who had not received neoadjuvant therapy were identified. After informed consent was obtained according to an institutional review board-approved protocol, a portion of each resected tumour was excised and mechanically dissociated and digested for 15-60 min with $1 \mathrm{mg} \mathrm{ml}^{-1}$ type II collagenase (Cell Isolation Optimizing System; Worthington Biochemical Corp., Lakewood, NJ, USA) in fresh $\mathrm{DMEM} / \mathrm{F} 12$, at $37^{\circ} \mathrm{C}$, all under sterile conditions. The cells were further dissociated using a gentleMACS tissue homogenizer (Miltenyi Biotec, Auburn, CA, USA). The resulting single-cell suspension was passed through a $100-\mu \mathrm{m}$ mesh filter and then resuspended in a 50:50 mixture of Hank's balanced salt solution and Cultrex basement membrane extract (Trevigen, Gaithersburg, MD, USA). This suspension was then injected subcutaneously into the flanks of athymic nude mice. The newly formed tumour was subsequently dissociated into a single-cell suspension; cells were co-stained for mouse major histocompatibility complex class I and human epithelial cell adhesion molecule (AbD Serotec, Oxford, $\mathrm{UK}$ ) and sorted by fluorescence-activated cell sorting (FACS) to minimise contamination by murine cells. The PDX-derived cells were used for sphere formation, tumorigenicity studies, or primary culture.
The primary CRC cell lines, HCP-1, HCP-2, HCP3-1, CC11-1, and $\mathrm{CC} 12-1$, were developed in our laboratory as described above. These cells are hereafter referred to collectively as 'early passage/ newly established CRC cell lines'. These five new cell lines, and the colorectal tumours from which they were derived were subjected to short-tandem repeat to ensure that they had the same genetic fingerprint as the matching patient tissues. Cell lines were cultured in MEM supplemented with $10 \%$ fetal bovine serum, penicillinstreptomycin, vitamins, sodium pyruvate, L-glutamine, and nonessential amino acids at $37^{\circ} \mathrm{C}$ in $5 \% \mathrm{CO}_{2}$ and $95 \%$ air. All in vitro experiments were performed at $60-80 \%$ confluence.

CD133 and CD44 FACS and Aldefluor assay. Samples were assessed using an Influx cell sorter (BD Biosciences, San Jose, CA, USA). Non-viable and non-epithelial cells were excluded from further analysis.

CD133 expression was assessed using anti-CD133/1phycoerythrin (Miltenyi Biotec), and CD44 was assessed using anti-CD44-fluorescein isothiocyanate (BD Pharmingen). Either mouse IgG-phycoerythrin (Miltenyi Biotec) or mouse IgG2b $\kappa$ fluorescein isothiocyanate isotype control (BD Pharmingen) was used as a control and gated at $\sim 0.1 \%$ out of 10000 events, cells in the top $5-10 \%$ of marker expression (high-expressing or positive cells) and cells in the lowest $5-10 \%$ of marker expression (low-expressing or negative cells) were analysed (Supplementary Figures 1 and 2); cells in the bottom 5\% of marker expression were eliminated to avoid collection of cellular debris. The flow cytometry data were analysed using FlowJo software version 7.6.5 (Tree Star, Ashland, OR, USA).

An Aldefluor kit (Stemcell Technologies, Vancouver, CA, USA) was used to identify cells with high ALDH enzymatic activity as previously described (Gaur et al, 2011). As a negative control, an aliquot from each sample was treated with $50 \mathrm{mmoll}^{-1}$ diethylaminobenzaldehyde, a specific ALDH inhibitor. DEAB was used as a negative control and gated at around $0.1 \%$ in vitro. Cells with high and low marker expression were selected as described in the preceding paragraph. Sham sorting (no addition of substrate) for viability and human markers were used to isolate a control population.

Sphere-forming assay. $\mathrm{CD}_{133^{+}}, \mathrm{CD}_{133^{-}}, \mathrm{CD} 44^{+}, \mathrm{CD} 44^{-}$, $\mathrm{ALDH}^{+}, \mathrm{ALDH}^{-}$, and sham-sorted cells were plated in 96-well, ultra-low-attachment plates (Corning Life Sciences, Lowell, MA, USA) at a density of 100 viable cells per well for established CRC cell lines and 5000 viable cells per well for the other models. Cells were grown in sphere medium consisting of DMEM/F12 (Invitrogen) supplemented with B27 serum-free supplement (1:50; Invitrogen), $20 \mathrm{ng} \mathrm{ml}^{-1}$ epidermal growth factor (R\&D Systems, Inc., Minneapolis, MN, USA), $20 \mathrm{ng} \mathrm{ml}^{-1}$ basic fibroblast growth factor (R\&D Systems, Inc.), and penicillin-streptomycin at $37^{\circ} \mathrm{C}$ in $5 \% \mathrm{CO}_{2}$. Fresh medium was added every 3-4 days, and the formation of free-floating spheres was monitored. The experiment was terminated after 7-21 days, and spheres were quantified ( $>50 \mu \mathrm{m}$ in diameter). The experiment was performed in triplicate.

For the serial sphere-forming assay, the cellular content of all triplicate wells (single cells and spheres combined) were harvested, pooled, and the spheres were dissociated with trypsin. Next, using trypan blue as a viability test, the resulting single CSC-enriched population was counted and 15000 viable cells were equally split into three new wells (5000 each) for the generation of tumour spheres.

In vivo serial tumorigenicity studies. Cells were sorted by FACS for each putative CSC marker (CD133, CD44, or ALDH activity). After sorting, cells were suspended in a 50:50 mixture of Hank's balanced salt solution and Cultrex basement membrane extract (Trevigen) and injected subcutaneously into the flanks of nude 
mice (10 mice per group) in a serial dilution assay (10 000 or 1000 for established cell lines, 5000 or 500 cells for freshly derived cell lines). Tumour growth was monitored three times a week with an endpoint of palpable tumours.

All of the first-passage tumour xenografts were resected when one of the xenografts reached $\sim 500 \mathrm{~mm}^{3}$. The tumours were digested and cells were re-sorted and injected for a second passage to study serial tumorigenicity. If first-passage tumours were not formed from a subgroup (e.g., CD44 - cells), then tumours formed from the sham-sorted cells were used to generate a second passage marker-negative tumour (e.g., $\mathrm{CD} 44^{-}$passage 2 ).

Statistical Analyses. For the in vitro studies, statistical analyses were done using Student's $t$-test (Microsoft Office Excel 2007, Redmond, WA, USA). For the in vivo studies, statistically significant difference of tumour incidence was calculated using Fischer's Exact Test. All statistical tests were two-sided, data represent means \pm s.e.m. and $P$-values $<0.05$ were considered statistically significant.

\section{RESULTS}

High CD44 expression does not enrich for cells with high sphere-forming capacity in established or freshly isolated CRC cell lines. The established human CRC cell lines such as HCT116, HT29, and SW480 have been broadly used in laboratory research. Several groups have used CD44 as a presumed surrogate marker for CSC-ness utilising these cell lines (Chu et al, 2009; Yeung et al, 2010). In order to determine whether the established human CRC cell lines can be used to identify CSCs by the putative CSC marker CD44, HCT116, HT29, and SW480 cells were sorted into CD44 ${ }^{+}$ and $\mathrm{CD} 44^{-}$by FACS and subjected to a sphere-forming assay. HCT116 cells formed significantly more spheres in the CD44 ${ }^{+}$ group than in the $\mathrm{CD} 44^{-}$group $(P<0.05)$. However, there was no difference in sphere formation between $\mathrm{CD} 44^{+}$and $\mathrm{CD} 44^{-}$

A

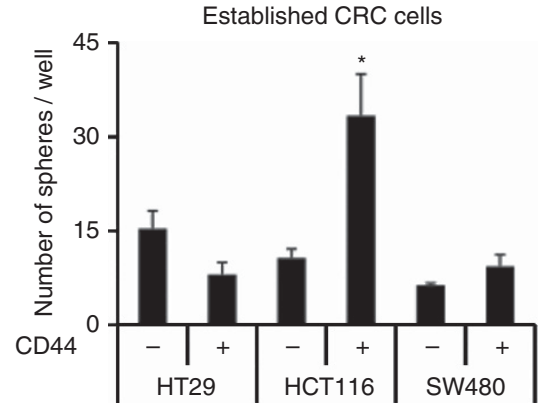

C

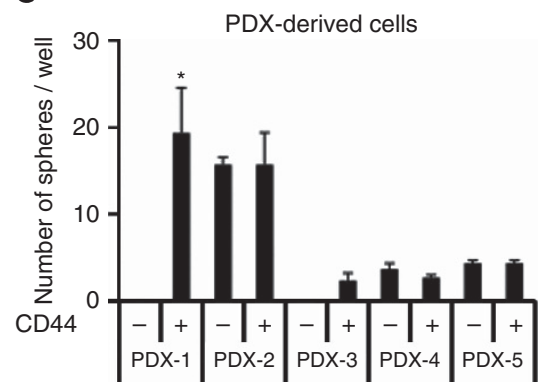

groups in HT29 and SW480 cells (Figure 1A), bringing into question the utility of CD44 as a universal CSC marker in CRC cells.

To determine whether the murine microenvironment can regenerate cellular heterogeneity and tumoural hierarchy from those homogeneous and highly passaged CRC cell lines, we used HCT116, HT29, and SW480 cells to create subcutaneous xenograft tumours in mice. These tumours were then resected, and the cells were sorted for $\mathrm{CD} 44^{+}$and $\mathrm{CD} 44^{-}$by FACS and subjected to sphere-forming assay. Results showed that sphere formation was not different between $\mathrm{CD} 44^{+}$and $\mathrm{CD} 44^{-}$cells in all three lines of xenografts tumours (Figure $1 \mathrm{~B}$ ) demonstrating that in vivo growth of established CRC cell lines does not restore cellular hierarchy and/or heterogeneity.

We next sought CD44 marker validation in PDX-derived cells. In PDX-1-derived cells, CD $44^{+}$cells formed significantly more spheres than CD44 - cells (Figure $1 \mathrm{C} ; P<0.001$ ). However, in the rest of PDXs $(-2,-3,-4,-5)$, high CD44 expression did not enrich for CSCs, again bringing into question the utility of CD44 as a universal CSC marker in CRC cells.

We next sought CD44 marker validation in early passage/newly established CRC cell lines, HCP-1, HCP2-1, HCP3-1, CC11-1, and CC12-1. The $\mathrm{CD}_{4} 4^{+}$- and $\mathrm{CD} 44^{-}$-sorted cells were subjected to sphere-forming assays. In HCP-1, CC11-1, and CC12-1 cells, high CD44-expressing cells formed significantly more spheres than CD44 low-expressing cells (Figures 1D, $P<0.01$ ). However, in HCP2-1 and HCP-3-1 cells, CD44 expression was not detected.

Taken together, these results further confirmed that CD44 cannot be used as a reliable CSC marker in CRC cells.

High CD133 expression does not consistently enrich for cells with high sphere-forming capacity in established or freshly isolated CRC cell lines. In an approach similar to that using CD44 marker, we studied the putative CSC marker CD133 in determining its utility in CSC isolation and validation. Sphereforming assays performed on HCT116, HT29, and SW480 showed
B

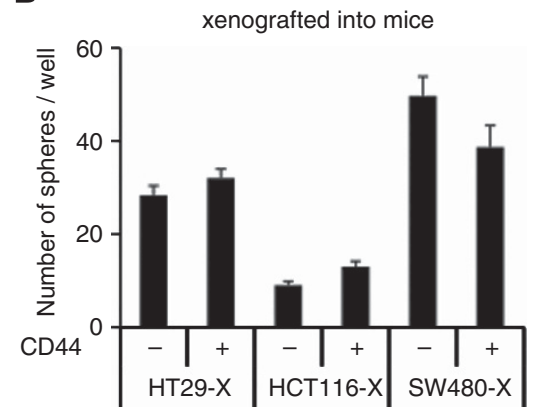

D

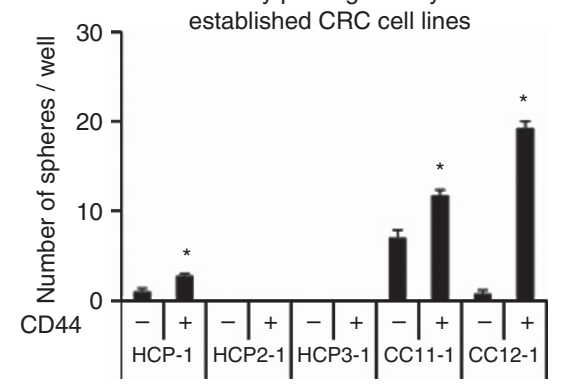

Figure 1. The utility of the cell surface markers CD44 to enrich for CSCs utilising the sphere-forming assay. Cells were sorted for CD44 expression in the following cellular models: (A) established CRC cell lines (HT29, HCT116, and SW480), (B) established CRC cells xenografted into mice (HT29-X, HCT116-X, and SW480-X), (C) PDXs (PDX-1 to 5) and (D) early passage/newly established CRC cell lines (HCP-1, HCP2-1, HCP3-1, CC11-1, and CC12-1). Sphere-forming assays were performed. Medians \pm s.e.m. are shown. 
A

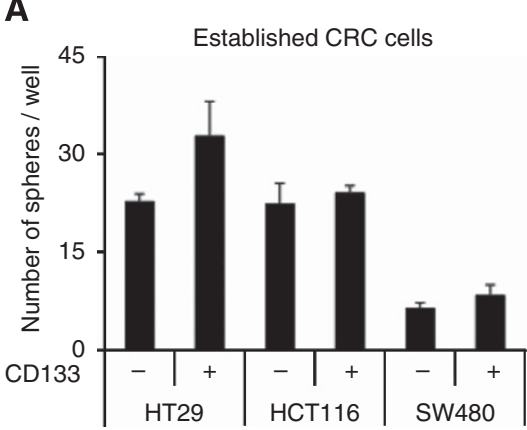

C

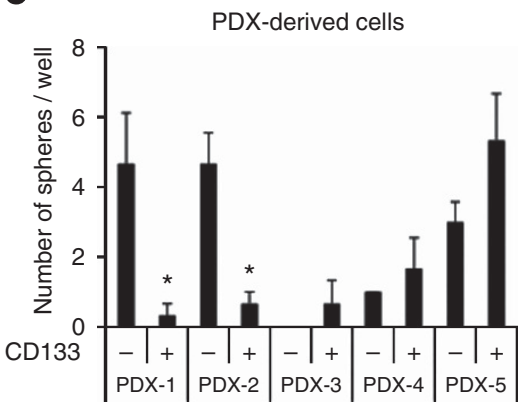

Established CRC cells
xenografted into mice

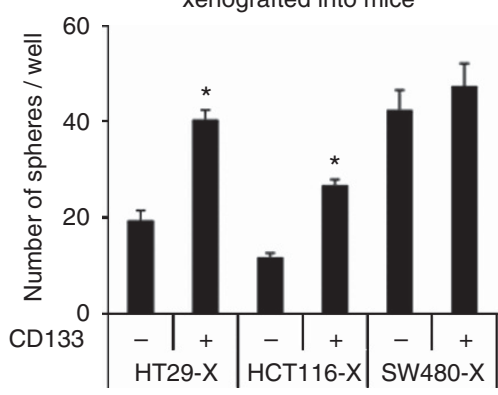

D

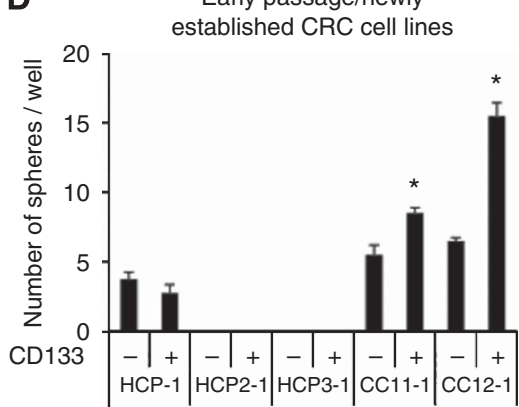

Figure 2. The utility of the cell surface marker CD133 to enrich for CSCs utilising the sphere-forming assay. Cells were sorted for CD133 expression in the following cellular models: (A) established CRC cell lines (HT29, HCT116 and SW480), (B) established CRC cells xenografted into mice (HT29-X, HCT116-X, and SW480-X), (C) PDXs (PDX-1 to 5) and (D) early passage/newly established CRC cell lines (HCP-1, HCP2-1, HCP3-1, CC11-1, and CC12-1). Sphere-forming assays were performed. Medians \pm s.e.m. are shown.

there was no significant difference in the sphere-forming capacity of $\mathrm{CD}_{133}{ }^{+}$or $\mathrm{CD}_{133}{ }^{-}$in all three CRC cell lines (Figure 2A). This brings into question the utility of established cell lines and CD133 as a marker of CSCs.

Interestingly, in HT29-X and HCT116-X cells isolated from their murine xenografted tumours, $\mathrm{CD}_{133^{+}}$subpopulations formed spheres at a significantly higher rate than CD133 - cells $(P<0.05)$. However, in SW480-X cells, CD133 ${ }^{+}$and $\mathrm{CD} 133^{-}$ cells did not significantly differ in sphere formation ability (Figure 2B). Therefore, the use of CD133 as a CSC marker for established CRC cell lines was not validated.

In PDX-1- and PDX-2-derived cells, CD133 ${ }^{+}$cells formed spheres less frequently than $\mathrm{CD}_{133^{-}}$cells $(P<0.001)$. However, there was no significant difference in the sphere-forming capacity between $\mathrm{CD}_{133^{+}}$and $\mathrm{CD} 133^{-}$subpopulations utilising PDX-3, -4 , and -5-derived cells (Figure 2C). This again brings into question the utility of CD133 as a marker of CRC stem-ness regardless of the cell type studied.

We next sought CD133 marker validation in early passage/ newly established CRC cell lines (five distinct cell lines). There were no significant differences in the sphere-forming capacity in HCP-1 cells sorted for high or low CD133 expression. In HCP2-1 and HCP3-1 cells, no CD133 expression was detected. In CC11-1 and CC12-1 cells, $\mathrm{CD}_{133^{+}}$cells formed more spheres compared with $\mathrm{CD}_{133^{-}}$cells $(P<0.02)$. This again brings into question the utility of CD133 as a marker of CRC stem-ness.

Finally, because of the seemingly promising results using CD133 as a CSC marker in HT29 and HCT116 cells after primary engraftment in mice (Figure 2B), but conflicting results using CD133 as a CSC marker in PDX-1- and PDX-2-derived cells (Figure 2C), we conducted further studies using in vivo dilutional tumorigenicity assays with $\mathrm{CD}_{13}{ }^{+}$and $\mathrm{CD} 133^{-} \mathrm{PDX}$-1-derived cells. Using PDX-1-derived cells, $\mathrm{CD}_{133^{+}}$cells yielded fewer tumours than $\mathrm{CD}_{133^{-}}$cells (Supplementary Table 1), suggesting that CD133 cannot be reliably used for enrichment of CSCs.
Taken together, these data demonstrate that CD133 is not a reliable CSC marker in CRC cells.

High ALDH activity enriches for cells with high sphere-forming capacity in freshly isolated but not established CRC cell lines. We next used ALDH activity-based Aldefluor assay to determine whether this method can be used to identify CSCs in established human CRC cell lines. SW480 cells with high ALDH activity formed significantly more spheres than low ALDH activity cells (Figure 3A; $P<0.05$ ). However, in HT29 and HCT116 cells, high or low ALDH activity could not reliably select for cells with increased sphere-forming capacity, demonstrating from the Aldefluor assay cannot select for CSCs from established cell lines growing in vitro.

In established CRC cells xenografted into mice, the results of sorting for $\mathrm{ALDH}^{+}$or $\mathrm{ALDH}^{-}$cells demonstrated inconsistencies in their sphere-forming capacity. HT29-X ALDH - cells formed slightly more spheres than $\mathrm{ALDH}^{+}$cells; there was no difference in sphere formation among HCT116-X and SW480-X ALDH ${ }^{+}$ and $\mathrm{ALDH}^{-}$cells (Figure $3 \mathrm{~B}$ ). These data confirm that the Aldefluor assay is not a valid method for isolating CSCs from established cell lines grown in vivo.

Next, we elected to assess CSC enrichment based on ALDH activity in our PDX-derived cells. $\mathrm{ALDH}^{+}$cells formed spheres at a significantly higher rate than the $\mathrm{ALDH}^{-}$population in PDX-1-derived cells (Figure 3C; $P<0.001$ ). Similar results were also shown in PDX-2, $-3,-4$, and -5 PDX-derived cells $(P<0.01)$. To determine that their sphere formation capacity was an intrinsic, rather than transient, feature of the $\mathrm{ALDH}^{+}$cell population, PDX-1-derived cells were further subjected to serial sphere formation assays. Results confirmed that the $\mathrm{ALDH}^{+}$cells maintained their CSC-ness throughout serial passage of spheres (Figure 3D; $P<0.001$ ). In contrast, the $\mathrm{ALDH}^{-}$cells formed only one sphere per well in the first passage but were unable to form spheres in secondary passage. As the salient feature of a true CSC 
A

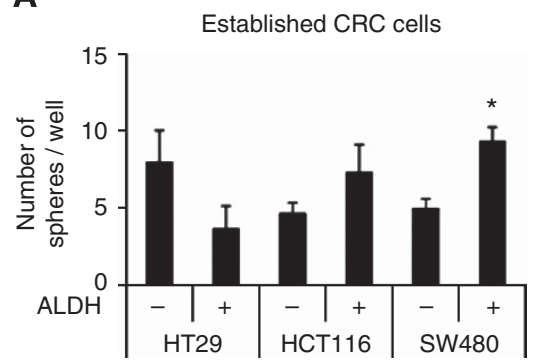

C

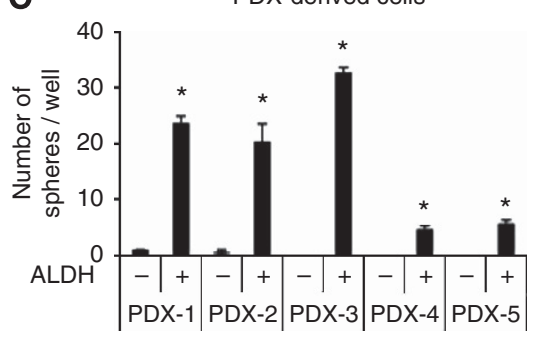

B Established CRC cells

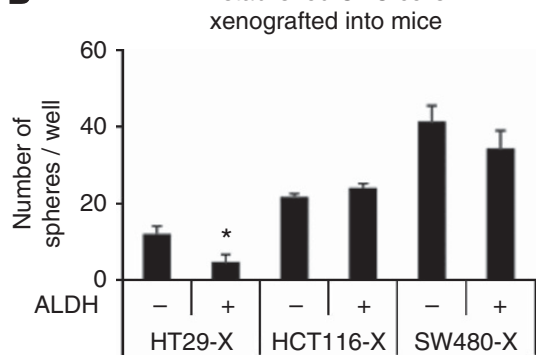

D

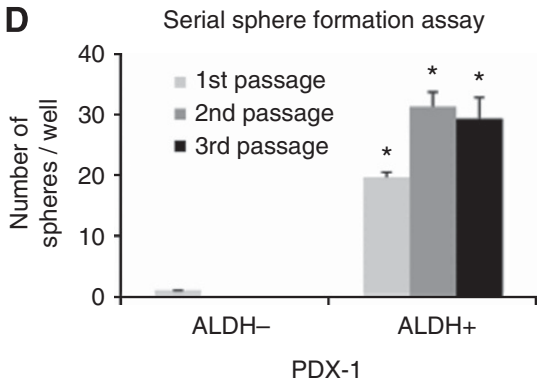

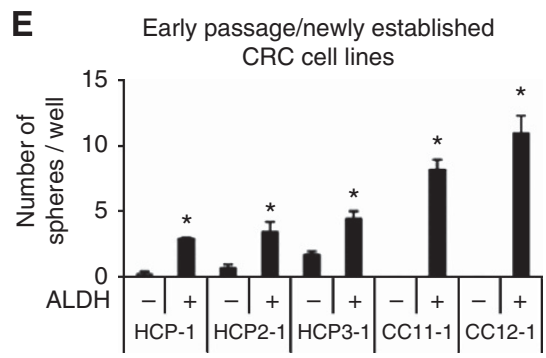

Figure 3. The utility of ALDH activity to enrich for CSCs utilising the sphere-forming assay. Cells were sorted for ALDH expression in the following cellular models: (A) established CRC cell lines (HT29, HCT116 and SW480), (B) established CRC cells xenografted into mice (HT29-X, HCT116-X, and SW480-X), (C) PDXs (PDX-1 to 5). Sphere-forming assays were performed. (D) Serial sphere-forming capacity in PDX-1-derived cells. (E) Sphere-forming assays were performed in early passage/newly established CRC cell lines, HCP-1, HCP2-1, HCP3-1, CC11-1, and CC12-1. Medians \pm s.e.m. are shown.

marker is also its negative predictive value (i.e., inability of the marker-negative cells to form spheres), $\mathrm{ALDH}^{-}$subpopulations of freshly isolated PDXs were indeed shown to exhibit very low basal activity in sphere formation, suggesting that ALDH is a reliable CSC marker for PDX-derived cells.

We next sought ALDH marker validation in early passage/newly established CRC cell lines, HCP-1, HCP2-1, HCP3-1, CC11-1, and CC12-1, and the results confirmed that $\mathrm{ALDH}^{+}$cells formed spheres at a significantly higher rate than the $\mathrm{ALDH}^{-}$population in all cell lines listed above (Figure 3E; $P<0.02$ ).

Taken together, these results showed that ALDH activity was not a reliable CSC marker for established cell lines, whereas ALDH activity was a reliable CSC marker when using newly developed established CRC cell lines.

High ALDH activity enriches for highly tumorigenic cells in PDX-derived cells, but not from established CRC cell lines. A critical experiment for determining the CSC phenotype is the in vivo tumorigenicity assay by serial dilution (Clarke et al, 2006; Lehmann et al, 2012). Therefore, we proceeded to confirm the ability of the Aldefluor assay to enrich for CSCs with in vivo serial dilutional tumorigenicity studies. $\mathrm{ALDH}^{+}, \mathrm{ALDH}^{-}$, and shamsorted populations from established CRC cell lines and PDX-1derived cells and were injected subcutaneously into nude mice. Using the established cell lines HT29, HCT116, and SW480, tumour incidence was similar regardless of the level of ALDH activity or the number of cells injected (Figure $4 \mathrm{~A}-\mathrm{C}$ ); this held true even after serial passages in vivo (data not shown). In contrast, the cell subpopulations from the PDX-1-derived cells when inoculating 5000 cells with high ALDH activity, eight out of eight animals reliably formed tumours $(P<0.05)$. This last observation held true with inoculums as small as 500 cells where the $\mathrm{ALDH}^{+}$ group formed tumours in four out of eight animals $(P<0.05)$. The subpopulation with low ALDH activity did not form tumours in either the 500- or 5000-cell group (Figure 4D).

Because the gold-standard assay for the CSC phenotype is both dilutional and serial tumorigenicity after primary passage in vivo, the tumours from $\mathrm{ALDH}^{+}$group were resected, dissociated and re-sorted for $\mathrm{ALDH}^{+}$cells. The cells were then re-injected into a second group of nude mice. As control, $\mathrm{ALDH}^{-}$cells were isolated from the primary tumours harvested in the sham group, as there were no primary tumours that formed in the $\mathrm{ALDH}^{-}$group in the first passage in mice. Result showed that $\mathrm{ALDH}^{+}$cells in the second passage formed tumours again (6 out of 10 mice), whereas $\mathrm{ALDH}^{-}$cells from the sham tumours were unable to form tumours (Supplementary Table 3).

Taken together, these studies support ALDH as a consistent and reliable marker of CRC-SCs derived from PDX-derived cells but not from established cell lines.

High ALDH activity reliably predicts sphere formation in cells isolated directly from a human tumour specimen. The previous studies were performed using established CRC cell lines in cell culture, established cell lines after in vivo passage, or the CRC PDX 
A

Established CRC cells tumourigenicity
\begin{tabular}{|c|c|c|}
\hline \multirow{2}{*}{ HT29 } & \multicolumn{2}{|c|}{ Inoculated cell number } \\
\cline { 2 - 3 } & 1000 & 10000 \\
\hline Sham & $10 / 10$ & $10 / 10$ \\
\hline ALDH- & $10 / 10$ & $10 / 10$ \\
\hline ALDH+ & $10 / 10$ & $10 / 10$ \\
\hline
\end{tabular}

B

\begin{tabular}{|c|c|c|}
\hline \multirow{2}{*}{ HCT116 } & \multicolumn{2}{|c|}{ Inoculated cell number } \\
\cline { 2 - 3 } & 1000 & 10000 \\
\hline Sham & $10 / 10$ & $10 / 10$ \\
\hline ALDH- & $10 / 10$ & $10 / 10$ \\
\hline ALDH+ & $10 / 10$ & $10 / 10$ \\
\hline
\end{tabular}

\begin{tabular}{|c|c|c|}
\hline \multirow{2}{*}{ SW480 } & \multicolumn{2}{|c|}{ Inoculated cell number } \\
\cline { 2 - 3 } & 1000 & 10000 \\
\hline Sham & $10 / 10$ & $10 / 10$ \\
\hline ALDH- & $10 / 10$ & $10 / 10$ \\
\hline ALDH+ & $10 / 10$ & $10 / 10$ \\
\hline
\end{tabular}

D

D PDX tumourigenicity
\begin{tabular}{|c|c|c|}
\hline \multirow{2}{*}{ PDX-1 } & \multicolumn{2}{|c|}{ Inoculated cell number } \\
\cline { 2 - 3 } & 500 & 5000 \\
\hline Sham & $1 / 8$ & $4 / 8$ \\
\hline ALDH- & $0 / 8$ & $0 / 8$ \\
\hline ALDH+ & $4 / 8$ & $8 / 8^{\star}$ \\
\hline
\end{tabular}

* Denotes statistically significant

defference between ALDH+ and ALDHcells (Fischer's exact test $P<0.05$ ).

Figure 4. ALDH activity reliably enriches for CSCs in vivo using in PDX-1-derived cells. (A-C) Established CRC cell lines, HT29, HCT116, and SW480, were $\mathrm{ALDH}^{+/-}$cell sorted and in vivo serial tumorigenicity studies were performed. (D) In vivo serial tumorigenicity studies in PDX-1derived cells.
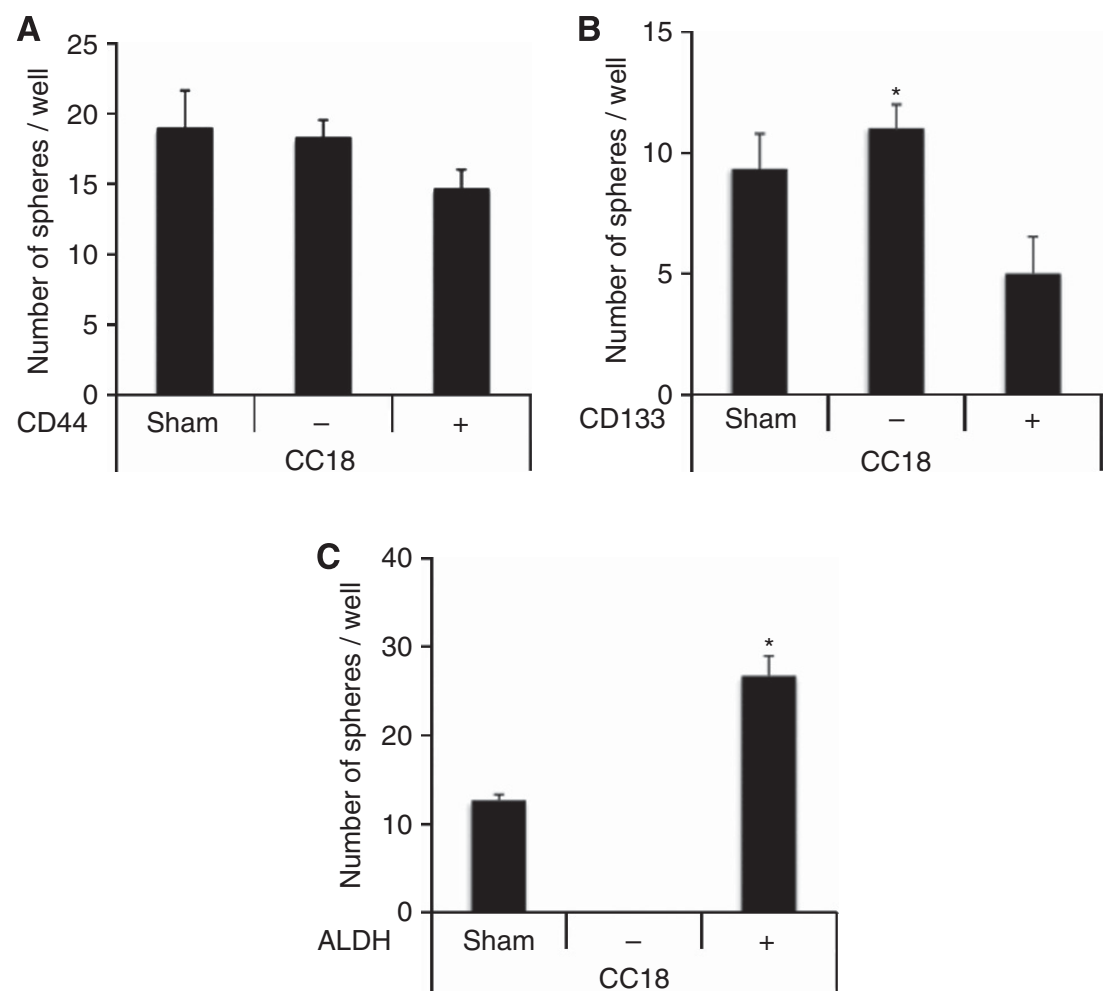

Figure 5. ALDH activity enriches for CSCs in CRC cells isolated directly from a patient tumour specimen. CRC cells isolated directly from a patient tumour specimen were sorted for (A) CD44 expression, (B) CD133 expression, and (C) ALDH activity.

cells that had been isolated after at least one passage of PDX in mice. To determine whether primary passage through mice affects the sphere-forming capacity of fresh surgical specimens, the cells isolated directly from patient tumour specimens were sorted by FACS into $\mathrm{CD}_{133^{+}}, \mathrm{CD}_{133^{-}}$; $\mathrm{CD} 44+, \mathrm{CD}_{4} 4^{-} ; \mathrm{ALDH}^{+}$,
$\mathrm{ALDH}^{-}$; and sham-sorted populations and were subjected to the sphere-forming assay. The results showed that both positive and negative populations of cells sorted by CD44 or CD133 FACS formed spheres regardless of their initial status (Figure 5A and B). Note that $\mathrm{CD}_{133}{ }^{+}$cells formed less spheres with fresh $\mathrm{CC} 18$ cells, 
which was similar to the observations with PDX-1- and PDX-2derived cells (Figure 2C), again demonstrating the lack of utility of CD133 as a CRC-SC marker. However, ALDH ${ }^{+}$CC18 cells formed significantly more spheres than those from sham-sorted cells and $\mathrm{ALDH}^{-}$cells (Figure 5C; $P<0.05$ ). The result was consistent with the observations made with three CRC PDX cells (Figure 3C) and confirmed that ALDH activity-based CSC enrichment was reproducible when cells were isolated from fresh surgical specimens. Owing to the limited size of the tumour specimen, there were only enough cells to perform sphere formation assays; tumorigenicity studies were not performed with these cells.

\section{DISCUSSION}

Isolation of a CSC-enriched population from CRC cells may provide new opportunities for target identification and drug development. In most studies reported in the literature, CSCs were isolated directly from human tumours after surgical resection (Dalerba et al, 2007; O'Brien et al, 2007; Ricci-Vitiani et al, 2007; Jung et al, 2011; Kondo et al, 2011). Although this method of isolation of CSCs may be preferred (and by most, considered the gold-standard), the logistics of using surgical samples for biologic studies warrants discussion. The use of human tumour specimens for CSC studies requires a coordinated team that can procure tumours efficiently and rapidly; it also requires a pathologist who can provide as large a tumour specimen as possible without affecting the primary pathologic evaluation. Tumour excision must be done in a timely manner because ischaemic time decreases cell viability and can alter protein expression (Gundisch et al, 2012; Neumeister et al, 2012). In fact, the 'clock' starts ticking in the operating room after devascularisation of the tumour and by the time a specimen is in the hands of the laboratory investigator, cell viability may be markedly diminished due to ischaemia, or the investigator may not acquire the tumour in time to be optimally studied. These issues are the pragmatic realities when investigators use fresh surgical specimens for the study of CSCs.

Our studies highlight two important findings to improve the ability to study CRC-SCs. First, one can use a small piece of tumour derived from a clinical specimen to perform a first passage through engraftment (PDX) in a mouse and then isolate CSCs from this xenografted tumour. The first passage through a mouse allows for tumour growth until there are sufficient tumour cells available for processing. This is an important step because CSCs represent only a small fraction of the tumour mass (Huang et al, 2009) and tumours can be processed under optimal conditions. The second major finding of our study is that the Aldefluor assay can be used to reliably enrich for CSCs from cells harvested from tumour xenografts, even after several passages of cells in culture following excision of the PDXs from mice. This method of identifying colorectal CSCs is much more efficient and reliable than isolating CSCs directly from surgical specimens, with the caveats and challenges outlined above.

It is important to delineate the reported role of established CRC cell lines in the study of CRC-SC biology. Although our data clearly do not support the use of established CRC cell lines for CSC studies, other investigators have reported on the use of established CRC cell lines for isolating CSCs (Ieta et al, 2008; Yeung et al, 2010). Yeung et al described the isolation of colorectal CSCs using the cell lines HT29 and SW1222, in which a population of CD44 ${ }^{+} /$ $\mathrm{CD}_{24}{ }^{+}$cells formed tumours in mice with a 200 -cell inoculum. However, they also reported that the $\mathrm{CD} 44^{-} / \mathrm{CD} 24^{-}$population formed tumours with inoculums of only 1000 cells. We studied SW1222 cells to determine if these cells could be used to identify a CRC-CS population. These studies showed that of all of the established cell lines, SW1222 was the cell line most amenable to
CSC isolation (Supplementary Figure 4). However, the inconsistencies in sphere formation and tumorigenicity in the CSC validation studies suggest that newly established cell lines or PDXderived cells remain the most reliable cell source in isolating CSCs, the main point of our manuscript.

Ieta et al (2008) described the use of CD133 in the established cell line HT29, but they characterised stemness using proliferation rate and invasion in vitro assays that are not widely used for CSC identification or enrichment. They also reported that injection of 5000 CD133 - cells into mice invariably yielded tumour growth, which agrees with our data (showing that CD133 as a marker of CSC-ness is not able to reliably identify CSCs; Ieta et al, 2008).

Unfortunately, few publications address the issue of the lack of utility of established CRC cell lines for CSC studies (Muraro et al, 2012). One typically learns of this issue by word of mouth after trying to use these cells for CSC studies, but failing to find cell populations that consistently follow the CSC model.

The use of the cell surface markers CD44 and CD133 to enrich for CSCs has had mixed results. We noted that the sphere-forming capacity of $\mathrm{CD}_{4} 4^{+}$HCT116 cells from in vitro culture was high, whereas it was low in the cells prepared from the HCT116 xenografted tumours (Figure 1A and B). This discrepancy validates our conclusion that CD44 should not be used as a CSC marker in CRC. Our studies showed that, when using cells cultured in vitro, the percentage of serum, cell density, and passage number were all parameters that were able to alter the CSC phenotype in both established CRC cell lines and freshly isolated CRC cell lines as shown in Supplementary Figure 3.

Contradictory data exist regarding the use of CD133 as a CSC marker, even when cells are isolated from clinical tumour specimens (Shmelkov et al, 2008; Kemper et al, 2010). In the studies utilising $\mathrm{CD} 133$ as a CRC-SC marker, $\mathrm{CD} 133^{+}$cells were not invariably tumorigenic, and CD133 - cells were noted to form spheres and generate tumours, although less frequently than $\mathrm{CD}_{133^{+}}$cells (O'Brien et al, 2007; Todaro et al, 2007; Lohberger et al, 2012). In our PDX model, the results paradoxically suggested that CD133 positivity could potentially be a negative CSC marker. Although this was not the focus of our study, this remains an interesting issue that would need to be further investigated in future studies. Similarly, data on CD44 as a CRC-SC marker are unclear. Some authors suggested that CD44 and CD133 expression are inversely related, whereas others suggested that these markers are co-expressed (Shmelkov et al, 2008; Vermeulen et al, 2008). Whether CD44 expression can identify CSCs alone or in conjunction with a second marker remains a point of controversy. CD24 and CD166 have been utilised as a second CSC marker for CRC-SCs, but further study is necessary to validate its use in enriching for the CSC phenotype (Dalerba et al, 2007; Todaro et al, 2007; Shmelkov et al, 2008; Vermeulen et al, 2008). Regardless, the CSC phenotype is a functional phenotype, and should only be validated by studies that identify this population in a functional assay(s).

The data presented in this manuscript demonstrate that it is possible to use PDX from freshly isolated tumour cells as an intermediate step for utilising surgical specimens to directly isolate a CRC cell population enriched for a CSC phenotype by Aldefluor staining and FAC sorting (summarised in Supplementary Table 2). This approach has yielded reproducible CSC phenotypic outcomes; CRC-SCs identified by this method can be further studied and characterised, providing a foundation for studies that may identify potential targets for novel therapeutic interventions.

\section{ACKNOWLEDGEMENTS}

We thank Sarah Bronson and Stephanie Deming from the Department of Scientific Publications and Rita Hernandez from 
the Department of Surgical Oncology at the University of Texas MD Anderson Cancer Center for editorial assistance.

This work was supported, in part, by National Institutes of Health (NIH) Cancer Center support grant CA016672, NIH grant T32CA009599 (to SB, FT, and ES), NIH Grant R01CA157880 (LME), DOD grant CA100879 (LME), the William C. Liedtke, Jr., Chair in Cancer Research (LME).

\section{REFERENCES}

Canino C, Mori F, Cambria A, Diamantini A, Germoni S, Alessandrini G, Borsellino G, Galati R, Battistini L, Blandino R, Facciolo F, Citro G, Strano S, Muti P, Blandino G, Cioce M (2012) SASP mediates chemoresistance and tumor-initiating-activity of mesothelioma cells. Oncogene 31(26): 3148-3163.

Chu P, Clanton DJ, Snipas TS, Lee J, Mitchell E, Nguyen ML, Hare E, Peach RJ (2009) Characterization of a subpopulation of colon cancer cells with stem cell-like properties. Int J Cancer 124(6): 1312-1321.

Clarke MF, Dick JE, Dirks PB, Eaves CJ, Jamieson CH, Jones DL, Visvader J, Weissman IL, Wahl GM (2006) Cancer stem cells-perspectives on current status and future directions: AACR Workshop on cancer stem cells. Cancer Res 66(19): 9339-9344.

Dalerba P, Dylla SJ, Park IK, Liu R, Wang X, Cho RW, Hoey T, Gurney A, Huang EH, Simeone DM, Shelton AA, Parmiani G, Castelli C, Clarke MF (2007) Phenotypic characterization of human colorectal cancer stem cells. Proc Natl Acad Sci USA 104(24): 10158-10163.

Du L, Wang H, He L, Zhang J, Ni B, Wang X, Jin H, Cahuzac N, Mehrpour M, $\mathrm{Lu} \mathrm{Y}$, Chen Q (2008) CD44 is of functional importance for colorectal cancer stem cells. Clin Cancer Res 14(21): 6751-6760.

Fredebohm J, Boettcher M, Eisen C, Gaida MM, Heller A, Keleg S, Tost J, Greulich-Bode KM, Hotz-Wagenblatt A, Lathrop M, Giese NA, Hoheisel JD (2012) Establishment and characterization of a highly tumourigenic and cancer stem cell enriched pancreatic cancer cell line as a well defined model system. PLoS One 7(11): e48503.

Gaur P, Sceusi EL, Samuel S, Xia L, Fan F, Zhou Y, Lu J, Tozzi F, Lopez-Berestein G, Vivas-Mejia P, Rashid A, Fleming JB, Abdalla EK, Curley SA, Vauthey JN, Sood AK, Yao JC, Ellis LM (2011) Identification of cancer stem cells in human gastrointestinal carcinoid and neuroendocrine tumors. Gastroenterology 141(5): 1728-1737.

Ginestier C, Hur MH, Charafe-Jauffret E, Monville F, Dutcher J, Brown M, Jacquemier J, Viens P, Kleer CG, Liu S, Schott A, Hayes D, Birnbaum D, Wicha MS, Dontu G (2007) ALDH1 is a marker of normal and malignant human mammary stem cells and a predictor of poor clinical outcome. Cell Stem Cell 1(5): 555-567.

Gundisch S, Hauck S, Sarioglu H, Schott C, Viertler C, Kap M, Schuster T, Reischauer B, Rosenberg R, Verhoef C, Mischinger HJ, Riegman P, Zatloukal K, Becker KF (2012) Variability of protein and phosphoprotein levels in clinical tissue specimens during the preanalytical phase. J Proteome Res 11(12): 5748-5762.

Huang EH, Hynes MJ, Zhang T, Ginestier C, Dontu G, Appelman H, Fields JZ, Wicha MS, Boman BM (2009) Aldehyde dehydrogenase 1 is a marker for normal and malignant human colonic stem cells (SC) and tracks SC overpopulation during colon tumorigenesis. Cancer Res 69(8): 3382-3389.

Hughes P, Marshall D, Reid Y, Parkes H, Gelber C (2007) The costs of using unauthenticated, over-passaged cell lines: how much more data do we need? Biotechniques 43(5): 575 577-8, 581-2 passim.

Ieta K, Tanaka F, Haraguchi N, Kita Y, Sakashita H, Mimori K, Matsumoto T, Inoue H, Kuwano H, Mori M (2008) Biological and genetic characteristics of tumor-initiating cells in colon cancer. Ann Surg Oncol 15(2): 638-648.

Jung P, Sato T, Merlos-Suarez A, Barriga FM, Iglesias M, Rossell D, Auer H, Gallardo M, Blasco MA, Sancho E, Clevers H, Batlle E (2011) Isolation and in vitro expansion of human colonic stem cells. Nat Med 17(10): 1225-1227.

Kemper K, Sprick MR, de Bree M, Scopelliti A, Vermeulen L, Hoek M, Zeilstra J, Pals ST, Mehmet H, Stassi G, Medema JP (2010) The AC133 epitope, but not the CD133 protein, is lost upon cancer stem cell differentiation. Cancer Res 70(2): 719-729.
Kim MP, Fleming JB, Wang H, Abbruzzese JL, Choi W, Kopetz S, McConkey DJ, Evans DB, Gallick GE (2011) ALDH activity selectively defines an enhanced tumor-initiating cell population relative to CD133 expression in human pancreatic adenocarcinoma. PLoS One 6(6): e20636.

Kondo J, Endo H, Okuyama H, Ishikawa O, Iishi H, Tsujii M, Ohue M, Inoue M (2011) Retaining cell-cell contact enables preparation and culture of spheroids composed of pure primary cancer cells from colorectal cancer. Proc Natl Acad Sci USA 108(15): 6235-6240.

Lehmann C, Jobs G, Thomas M, Burtscher H, Kubbies M (2012) Established breast cancer stem cell markers do not correlate with in vivo tumorigenicity of tumor-initiating cells. Int J Oncol 41(6): 1932-1942.

Lohberger B, Rinner B, Stuendl N, Absenger M, Liegl-Atzwanger B, Walzer SM, Windhager R, Leithner A (2012) Aldehyde dehydrogenase 1, a potential marker for cancer stem cells in human sarcoma. PLoS One 7(8): e43664.

Moreb JS, Ucar D, Han S, Amory JK, Goldstein AS, Ostmark B, Chang LJ (2012) The enzymatic activity of human aldehyde dehydrogenases $1 \mathrm{~A} 2$ and 2 (ALDH1A2 and ALDH2) is detected by Aldefluor, inhibited by diethylaminobenzaldehyde and has significant effects on cell proliferation and drug resistance. Chem Biol Interact 195(1): 52-60.

Muraro MG, Mele V, Daster S, Han J, Heberer M, Cesare Spagnoli G, Iezzi G (2012) CD133 +, CD166 + CD44 +, and CD24 + CD44 + phenotypes fail to reliably identify cell populations with cancer stem cell functional features in established human colorectal cancer cell lines. Stem Cells Transl Med 1(8): 592-603.

Neumeister VM, Anagnostou V, Siddiqui S, England AM, Zarrella ER, Vassilakopoulou M, Parisi F, Kluger Y, Hicks DG, Rimm DL (2012) Quantitative assessment of effect of preanalytic cold ischemic time on protein expression in breast cancer tissues. J Natl Cancer Inst 104(23): 1815-1824.

O’Brien CA, Pollett A, Gallinger S, Dick JE (2007) A human colon cancer cell capable of initiating tumour growth in immunodeficient mice. Nature 445(7123): 106-110.

Ricci-Vitiani L, Lombardi DG, Pilozzi E, Biffoni M, Todaro M, Peschle C, De Maria R (2007) Identification and expansion of human colon-cancerinitiating cells. Nature 445(7123): 111-115.

Shmelkov SV, Butler JM, Hooper AT, Hormigo A, Kushner J, Milde St T, Clair R, Baljevic M, White I, Jin DK, Chadburn A, Murphy AJ, Valenzuela DM, Gale NW, Thurston G, Yancopoulos GD, D’Angelica M, Kemeny N, Lyden D, Rafii S (2008) CD133 expression is not restricted to stem cells, and both CD133 + and CD133 - metastatic colon cancer cells initiate tumors. J Clin Invest 118(6): 2111-2120.

Storms RW, Trujillo AP, Springer JB, Shah L, Colvin OM, Ludeman SM, Smith C (1999) Isolation of primitive human hematopoietic progenitors on the basis of aldehyde dehydrogenase activity. Proc Natl Acad Sci USA 96(16): 9118-9123.

Todaro M, Alea MP, Di Stefano AB, Cammareri P, Vermeulen L, Iovino F, Tripodo C, Russo A, Gulotta G, Medema JP, Stassi G (2007) Colon cancer stem cells dictate tumor growth and resist cell death by production of interleukin-4. Cell Stem Cell 1(4): 389-402.

Vermeulen L, Todaro M, de Sousa Mello F, Sprick MR, Kemper K, Perez Alea M, Richel DJ, Stassi G, Medema JP (2008) Single-cell cloning of colon cancer stem cells reveals a multi-lineage differentiation capacity. Proc Natl Acad Sci USA 105(36): 13427-13432.

Wu H, Zhang H, Hu Y, Xia Q, Liu C, Li Y, Yu B, Gu T, Zhang X, Yu X, Kong W (2014) Sphere formation assay is not an effective method for cancer stem cell derivation and characterization from the Caco-2 colorectal cell line. Curr Stem Cell Res Ther 9(2): 82-88.

Yeung TM, Gandhi SC, Wilding JL, Muschel R, Bodmer WF (2010) Cancer stem cells from colorectal cancer-derived cell lines. Proc Natl Acad Sci USA 107(8): 3722-3727.

This work is published under the standard license to publish agreement. After 12 months the work will become freely available and the license terms will switch to a Creative Commons AttributionNonCommercial-Share Alike 3.0 Unported License.

Supplementary Information accompanies this paper on British Journal of Cancer website (http://www.nature.com/bjc) 